Second, human osteoblasts $(\mathrm{Ob})$ were obtained from post-surgery discarded trabecular bone of osteoarthritic (OA) patients who underwent total knee arthroplasty. First passage human OA Ob were treated either with RvD1 (0.1 $1 \mu \mathrm{M})$ alone, or with $20 \mathrm{nM}$ VitD3 with or without RvD1 $(0.1-1 \mu \mathrm{M})$, for 48 hours. Cell viability was evaluated with the MTS test. Alkaline phosphatase (PAL) activity and osteocalcin (OCN) release was determined by colorimetric reaction and ELISA, respectively.

Results: In RAW264.7 cells, our results clearly show that RvD1 strongly reduces OC recruitment and activation as indicated by the inhibition of TRAP and cathepsin $\mathrm{K}$ expression as well as TNF- $\alpha, \mathrm{IL}-1 \beta, \mathrm{IL}-6, \mathrm{PGE}_{2}$ and NO release, as well as the concurrent enhancement of IL-10 levels. Besides, RvD1 decreases bone resorption through the inhibition of plots formation in hydroxyapatite matrix. In human OA Ob, RvD1 partially decreases VitD3-induced PAL activity, while it maintains $O C N$ expression at control levels.

Conclusions: Our in vitro results clearly show that RvD1 may play an important role in the regulation of bone metabolism. Additionally to our previous data, our findings suggest that RvD1 may offer a novel and original perspective to make a real contribution to musculoskeletal and bone diseases therapy.

Acknowledgements: This study was supported by Canadian Institute of Health Research (CIHR) grant (\#IMH 131570) and by the Center of Excellence for Arthroplasty Research.

Disclosure of Interest: None declared

DOI: 10.1136/annrheumdis-2017-eular.2934

\section{THU0062 NOVEL ANIMAL MODEL OF AROMATASE INHIBITOR-INDUCED ARTHRALGIA SUGGESTS AN ESTROGEN-INDEPENDENT INFLAMMATORY MECHANISM}

N.A. Young ${ }^{1}$, E. Thomas ${ }^{1}$, B. Snoad ${ }^{1}$, J. Sharma ${ }^{1}$, M. Mobeen ${ }^{1}$, A.C. DeVries ${ }^{2}$, A. Bratasz ${ }^{3}$, M. Lustberg ${ }^{4}$, W. Jarjour ${ }^{1}$, R. Reinbolt ${ }^{4}$. ${ }^{1}$ Rheumatology and Immunology; ${ }^{2}$ Department of Neuroscience; ${ }^{3}$ Small Animal Imaging Core; ${ }^{4}$ Medical Oncology, The Ohio State University Wexner Medical Center, Columbus, United States

Background: Aromatase Inhibitors (Als) block physiological estrogen production in peripheral tissues and significantly improve overall survival rates of postmenopausal, hormone receptor-positive breast cancer patients by reducing tumor recurrences. However, half of patients taking these drugs develop aromatase inhibitor induced arthralgia (AllA), which is characterized by severe pain and inflammation in various joints. Since AllA leads to suspension of therapy in $20 \%$ of patients, reducing incidence may provide sustained Al treatment and enhanced long-term survival.

Objectives: In order to establish a better understanding of the inflammatory mechanism and to create a platform that can be used to explore interventional strategies, our objective in this study was to design a novel animal model of AllA. Methods: Female BALB/C-Tg (NFKB-RE-luc)-Xen mice, which have a firefly luciferase $\mathrm{CDNA}$ reporter gene under the regulation of $3 \mathrm{kB}$ responsive binding sites, were oophorectomized and treated with $\mathrm{Al}$ (letrozole) by daily subcutaneous injections. Control groups included oophorectomized mice receiving vehicle control injections and non-oophorectomized mice treated with Al. Bioluminescent imaging of hind limbs was performed after 3 weeks on the in vivo imaging system (IVIS) to measure NFKB activation. At 5 weeks, knee joints and surrounding tissue were imaged on the BioSpec 94/30 micro-MRI. Legs were collected for histopathological analysis and serum cytokine levels were measured at experimental endpoint.

Results: Bioluminescent imaging showed significantly enhanced NFKB activation in the hind limbs compared to oophorectomized controls receiving vehicle treatment. Analysis of knee joints and legs by MRI imaging showed enhanced signal detection in the joint space and surrounding tissue following Al treatment. Surprisingly, enhanced MRI detection was also demonstrated in non-oophorectomized mice that were treated with Al. Histopathological analysis further demonstrated mild inflammation in the synovial tissue and joint damage in mice treated receiving Al both with and without oophorectomy. Moreover, tenosynovitis and inflammatory muscle tissue infiltrates were detected in Al-treated mice and serum cytokine levels of IL-2, IL-4, IL-6, and CXCL1 were significantly elevated.

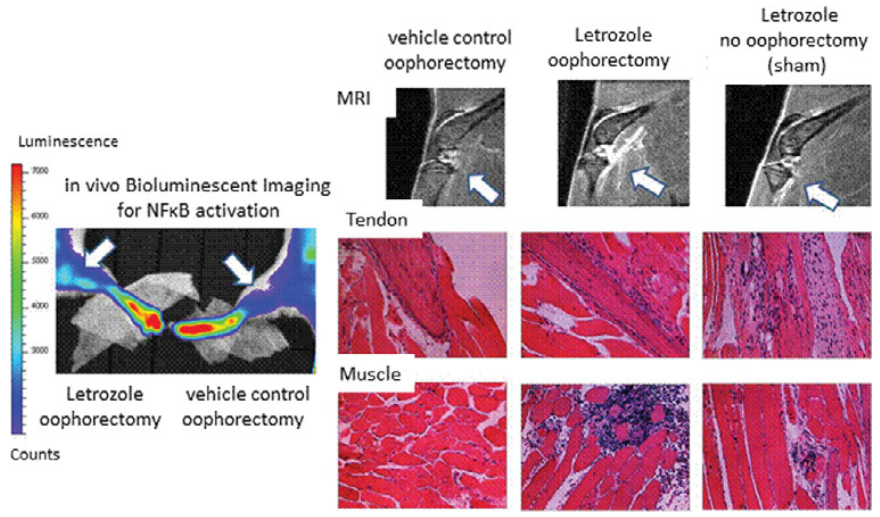

Conclusions: Collectively, these data establish a novel mouse model of AllA and suggest that the pathogenesis of Al-induced inflammation is estrogenindependent. Future studies will be directed into the characterization of this inflammatory mechanism to provide insight into potential therapeutic strategies directed at mitigating this adverse inflammatory burden.

References:

[1] Henry NL, Giles JT, Ang D, et al. Prospective characterization of musculoskeletal symptoms in early stage breast cancer patients treated with aromatase inhibitors. Breast cancer research and treatment. 2008:111(2):365-372.

[2] Mao JJ, Stricker C, Bruner D, et al. Patterns and risk factors associated with aromatase inhibitor-related arthralgia among breast cancer survivors. Cancer. 2009;115(16):3631-3639.

Acknowledgements: Stefanie Spielman Fund for Breast Cancer Research and The Wexner Medical Center.

Disclosure of Interest: None declared

DOI: 10.1136/annrheumdis-2017-eular.5424

\section{THU0063 IL-1 FAMILY CYTOKINES AND RECEPTORS IN IGG4-RELATED DISEASE}

R. Capecchi ${ }^{1}$, P. Italiani ${ }^{2}$, I. Puxeddu ${ }^{1}$, F. Pratesi ${ }^{1}$, A. Tavoni ${ }^{3}$, D. Boraschi ${ }^{2}$, P. Migliorini ${ }^{1} .{ }^{1}$ Clinical and Experimental Medicine, Clinical Immunology Unit, university of Pisa, pisa; ${ }^{2}$ Institute of Protein Biochemistry, National Research Council, Naples; ${ }^{3}$ Clinical and Experimental Medicine, University of Pisa, Clinical Immunology Unit, university of Pisa, pisa, Italy

Background: IgG4-related disease (IgG4-RD) is a fibroinflammatory condition that can affect almost any organ, characterized by lymphoplasmocytoid infiltrate, obliterative phlebitis and storiform fibrosis often associated with eosinophilia and increased levels of IgG4. Cytotoxic CD4 T cells producing IL-1b [D1], TGFb1 and IFN-gare detectable in peripheral blood of patients and high IL-18 expression has been found in affected organs.

Objectives: To evaluate the role of IL-1 family cytokines in IgG4-RD, by analyzing cytokines and receptors in sera.

Methods: Nine patients fulfilling the proposed criteria (Umehara, 2012) for the diagnosis of IgG4-RD were recruited. Cytokines of the IL-1 family (IL-1a, IL-1b, IL-33, IL-18), soluble receptors (sIL-1R1, sIL-1R2, sIL-1R3, sIL-1R4) and antagonists (IL-1Ra, IL-18BP) were measured in sera by multiarray ELISA assay. Free IL-18 was calculated using the law of mass action.

Results: Most patients had a multiorgan disease; retroperitoneum, salivary glands, pancreas and lymph nodes were most frequently affected. IL-18 ( $p=0.007)$ and free IL-18 $(p<0.0001)$, slL-1R1 $(p=0.0005)$, sIL-1R2 $(p=0.0013)$, and sIL-1R4 $(p=0.0006)$ were significantly increased in IgG4-RD sera compared with healthy controls.

Conclusions: In IgG4-RD patients, at variance with other autoimmune or autoinflammatory conditions, the increase in IL-18 levels is not counterbalanced by IL-18BP, leading to high levels of free IL-18. The free cytokine may affect T cell subset balance and induce IFN-g production. The parallel increase of SIL-1R1 and sIL-1R2 suggests an efficient dampening of inflammatory IL-1bsignaling at the tissue level, while high levels of SIL-1R4 may be associated with vascular remodeling and fibrosis, as observed in animal models of obesity and in human cardiovascular disorders.

Disclosure of Interest: None declared

DOI: 10.1136/annrheumdis-2017-eular.5816

\section{THU0064 ADAM-10 AS A TOCILIZUMAB TREATMENT PREDICTIVE} FACTOR IN RHEUMATOID ARTHRITIS

T. Isozaki, S. Nishimi, A. Nishimi, S. Ishii, T. Tokunaga, H. Furuya,

K. Wakabayashi, T. Kasama. Division of Rheumatology, Department of Medicine, Showa University School of Medicine, Tokyo, Japan

Background: A disintegrin and metalloproteinases (ADAMs) are a family of transmembrane and secreted proteins. ADAM-10 has been reported to be the enzyme responsible for the release of a number of chemokines and cytokine receptors. We have shown that ADAM-10 is overexpressed on rheumatoid arthritis (RA) synovial tissue endothelial cells (ECs) and lining cells compared with osteoarthritis and normal tissues. We also demonstrated that ADAM-10 mediates EC migration and tube formed.

Objectives: In order to demonstrate for ADAM-10 in clinical side, we focused on ADAM-10 as predictive factor for treatment with biologics in RA.

Methods: The serum was collected from patients before the initial treatment with biological therapies. Fifteen patients were treated with adalimumab (ADA), and 20 patents were treated with tocilizumab (TCZ). ADAM-10 and fractalkine/CX3CL1 were measured by enzyme-linked immunosorbent assay at 0,12, 24 and 54 weeks. Clinical disease activity was evaluated by clinical disease activity index (CDAI). Following biological therapies, we defined biologic-responders as patients whose DAS28 scores decreased by more than 1.2 at 24 weeks. ADAM-10 baseline was also compared between responders and nonresponders at 24 weeks.

Results: There were no significant differences were observed in the mean age, gender ratio, dosages of predonisolone and methotraxate between ADA and TCZ groups. In ADA group, baseline DAS28 for the 15 patients was $4.8 \pm 0.3(2.5-7.2)$. 